\title{
Solar Wind Sources in the Late Declining Phase of Cycle 23: Effects of the Weak Solar Polar Field on High Speed Streams
}

\author{
J.G. Luhmann • C.O. Lee $\cdot$ Yan Li · C.N. Arge • \\ A.B. Galvin · K. Simunac · C.T. Russell • R.A. Howard • \\ G. Petrie
}

Received: 4 December 2008 / Accepted: 1 April 2009 / Published online: 21 April 2009

(C) The Author(s) 2009. This article is published with open access at Springerlink.com

\begin{abstract}
The declining phases of solar cycles are known for their high speed solar wind streams that dominate the geomagnetic responses during this period. Outstanding questions about these streams, which can provide the fastest winds of the solar cycle, concern their solar origins, persistence, and predictability. The declining phase of cycle 23 has lasted significantly longer than the corresponding phases of the previous two cycles. Solar magne-
\end{abstract}

STEREO Science Results at Solar Minimum

Guest Editors: Eric R. Christian, Michael L. Kaiser, Therese A. Kucera, O.C. St. Cyr.

J.G. Luhmann $(\bowtie) \cdot$ C.O. Lee · Y. Li

Space Sciences Laboratory, University of California, Berkeley, CA 94720, USA

e-mail: jgluhman@ssl.berkeley.edu

C.N. Arge

AFRL/VSBXS, 29 Randolph Road, Hanscom AFB, Boston, MA 01731, USA

e-mail: nick.arge@ hanscom.af.mil

A.B. Galvin · K. Simunac

Space Science Center, University of New Hampshire, Morse Hall, Durham, NH 03824, USA

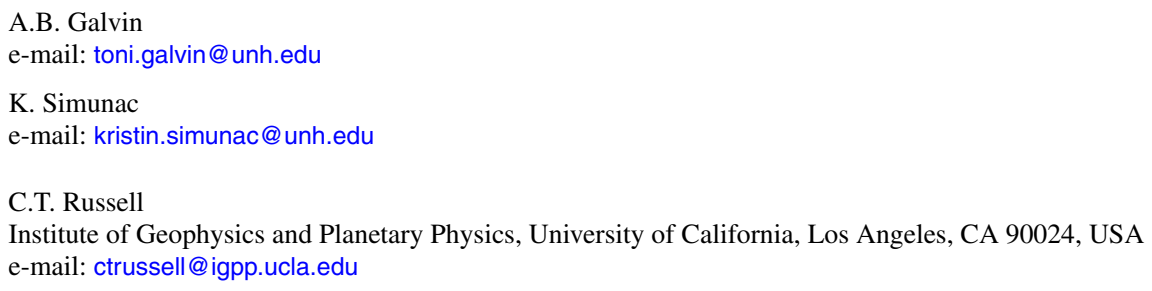

R.A. Howard

Solar Physics Branch, Naval Research Laboratory, 4555 Overlook Ave SW, Washington, DC 20375,

USA

e-mail: russ.howard@nrl.navy.mil

G. Petrie

National Solar Observatory, 950 N Cherry Ave, Tucson, AZ 85719, USA

e-mail: gpetrie@nso.edu 
tograph observations suggest that the solar polar magnetic field is also $\sim 2-3$ times weaker. The launch of STEREO in late 2006 provided additional incentive to examine the origins of what is observed at $1 \mathrm{AU}$ in the recent cycle, with the OMNI data base at the NSSDC available as an Earth/L1 baseline for comparisons. Here we focus on the year 2007 when the solar corona exhibited large, long-lived mid-to-low latitude coronal holes and polar hole extensions observed by both SOHO and STEREO imagers. STEREO provides in situ measurements consistent with rigidly corotating solar wind stream structure at up to $\sim 45^{\circ}$ heliolongitude separation by late 2007. This stability justifies the use of magnetogram-based steady $3 \mathrm{D}$ solar wind models to map the observed high speed winds back to their coronal sources. We apply the WSA solar wind model currently running at the NOAA Space Weather Prediction Center with the expectation that it should perform its best at this quiet time. The model comparisons confirm the origins of the observed high speed streams expected from the solar images, but also reveal uncertainties in the solar wind source mapping associated with this cycle's weaker solar polar fields. Overall, the results illustrate the importance of having accurate polar fields in synoptic maps used in solar wind forecast models. At the most fundamental level, they demonstrate the control of the solar polar fields over the high speed wind sources, and thus one specific connection between the solar dynamo and the solar wind character.

Keywords STEREO mission $\cdot$ Solar wind $\cdot$ Solar cycle $\cdot$ Solar magnetic field $\cdot$ PFSS model

\section{Introduction}

The launch of the Solar Terrestrial Relations Observatory (STEREO) twin spacecraft in October 2006 provided a new set of multipoint solar wind measurements that can be used to understand the coronal sources of the solar wind, at a time when it is expected to be exceptionally stable and traceable. Previous observations of $\sim 27$ day periodicities in interplanetary plasma and field features during the declining phases of solar cycles have suggested that slowly evolving conditions of the coronal field topology produce a nearly rigidly corotating solar wind structure at these times. The quasi-steady conditions occur in spite of the continuing emergence of some late cycle active regions and the ongoing redistribution and decay of previously emerged photospheric magnetic fields. From a space weather perspective, the associated corotating high speed streams are known to underlie enhancements in the Earth's relativistic magnetospheric electron populations, and recurrent small to moderate geomagnetic storms through the stream interaction regions they produce (e.g. Baker et al., 1997).

Since late January 2007 the STEREO spacecraft have been separating from one another at a rate of $\sim 45^{\circ}$ year $^{-1}$. This has allowed us to observe the near-ecliptic solar wind at $1 \mathrm{AU}$ at increasing distances from the upstream (L1) location where conditions affecting Earth are routinely monitored. Here we focus on a $\sim 10$ month period from the first year of STEREO measurements, with the OMNI data base (King and Papatashvilii, 1994) providing L1 comparisons, toward understanding the character of the late declining phase solar wind.

Of particular interest in this study are the effects of the weak solar polar magnetic fields of this cycle on the solar wind structure. Two recent papers describe some apparent differences from the previous cycle declining phase seen in Ulysses measurements of the interplanetary magnetic field (Smith and Balogh, 2008) and the solar wind mass flux (McComas et al., 2008). An accompanying paper by Lee et al. (2009) investigates the nature of the weaker interplanetary magnetic field seen in the ecliptic this cycle. The present analysis considers the complementary subject of the sources of the related high speed streams and the possible influence of the weak solar polar fields. 
We first show the STEREO Ahead and Behind solar wind and interplanetary field observations from 10 Carrington Rotations (2052-2062) in 2007 together with the OMNI L1 data. Their similarities illustrate how well the assumption of steady corotating structure is adhered to over baselines of up to $\sim 45^{\circ}$ (also see Simunac et al., 2009), justifying the use of steady models of the corona and solar wind to interpret the data. We then apply the solar magnetogram-based potential field source surface (PFSS) model (e.g. Altschuler and Newkirk, 1969) to investigate the inferred coronal open field sources of the high speed streams. The WSA (Wang-Sheeley-Arge) coupled PFSS and steady solar wind model (Arge and Pizzo, 2000), based on daily updating solar magnetic field synoptic maps, is then used to map the outflows from the corona to 1 AU. The comparisons with in situ observations reveal features of both the solar wind sources and the models. In particular they illustrate the challenges faced by models even at times when the solar wind is ostensibly at its best-behaved phase, as well as the control of the solar polar magnetic field over the sources that produce the high speed winds.

\section{STEREO and OMNI Observations of the Solar Wind and Its Sources}

The STEREO observations described here were obtained from February through October 2007, when the STEREO Ahead (-A) and Behind (-B) spacecraft were in their heliocentric orbits between the positions shown in Figures 1(a) and (b). Corresponding OMNI data were obtained from Advanced Composition Explorer (ACE) and WIND near the L1 point, approximately midway between the two STEREO spacecraft. The heliolongitude separation of each STEREO spacecraft from the Earth increases approximately $22.5^{\circ}$ year $^{-1}$. Although the STEREO spacecraft deviate slightly from 1.0 AU and from the ecliptic plane, these deviations are considered negligible for the purposes of this study of large scale features. As mentioned above, the period investigated corresponds to Carrington Rotations (CR) 2052 to 2062. Only a few Earth-directed coronal mass ejections (CMEs) with detected ICMEs (Interplanetary CMEs) occurred during this time, the most significant in the third week of May during CR 2056 (see Li et al., 2008; Kilpua et al., 2009).

Two investigations on STEREO provide the main in situ solar wind observations: Plasma and Suprathermal Ion Composition (PLASTIC) (Galvin et al., 2008) measures the plasma ion moments, while the In situ Measurements of Particles and CME Transients (IMPACT) (Luhmann et al., 2008) measures the interplanetary magnetic field and the plasma electrons. The STEREO data are available through website links at the Stereo Science Center (http://stereo-ssc.nascom.nasa.gov), while the OMNI data can be downloaded from the National Space Science Data Center (http://omniweb.gsfc.nasa.gov). The OMNI plasma moments for this period are based either on ACE SWEPAM or WIND SWE measurements (described by McComas et al., 1998 and Ogilvie et al., 1995, respectively), with the magnetic fields mainly from the ACE magnetometer (e.g. Smith et al., 1998). For the present study the ion velocity and magnetic field polarity are the primary quantities used for source mapping.

Figures 2(a-c) provide an overview of the STEREO-A and B observations of the magnetic field magnitude and radial component at 10 minute resolution, and the plasma bulk velocity, compared to the corresponding hourly OMNI data. Carrington Rotations are separated by vertical lines. The velocities (Figure 2(c)) show high speed streams with typical maximum speeds of $500-700 \mathrm{~km} \mathrm{~s}^{-1}$ lasting several Carrington Rotations at a time. The magnetic field magnitude and radial components in Figures 2(a) and (b) have features consistent with stream interaction regions, some of which contain the polarity reversals of the 
Figure 1 Positions of the STEREO spacecraft relative to Earth/ACE in early February (a - upper panels) and early December ( $b$ - lower panels). The letters A and B identify the locations of the STEREO-A and STEREO-B spacecraft, respectively. The ACE spacecraft is effectively at the position of the Earth (green dot) on this scale.
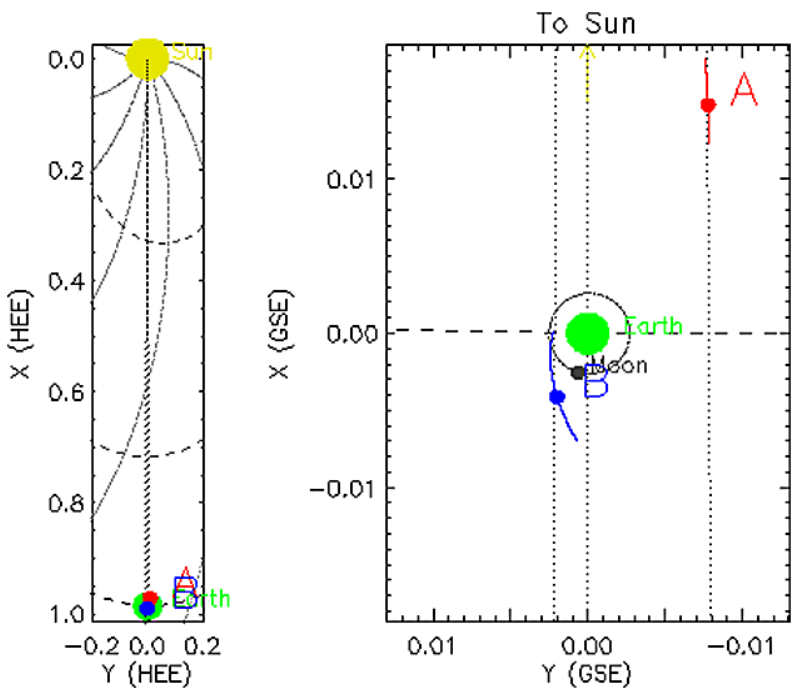

(a)

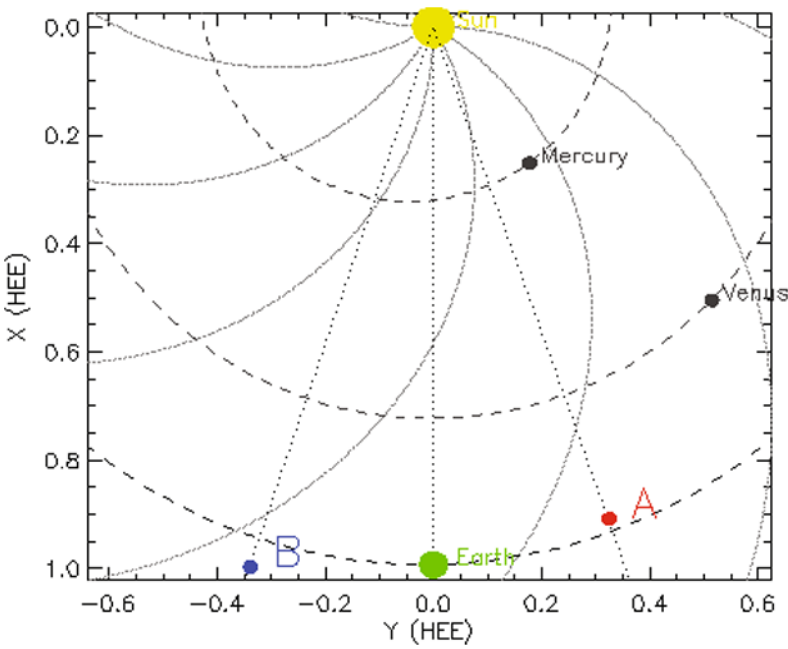

(b)

heliospheric current sheet (e.g. Gosling et al., 1978). These observations reinforce the idea that when solar activity is low, the solar wind evolves in a quasi-steady manner, such that particular streams can be followed, sometimes for months, at a fixed heliospheric location, They can also be recognized at other heliospheric longitudes if appropriate corotation lead times or delays are taken into account (e.g. Simunac et al., 2009). This slow evolution is key to the ability to use models for source identification/mapping as described below.

The prevailing solar sources of the solar wind observed by STEREO and at L1 in 2007 are seen in the EUV images from SOHO EIT (Delaboudiniere et al., 1997) and STEREO SECCHI-EUVI (Howard et al., 2008) in Figure 3. The images in the top row show nearly identical disks observed when the STEREO spacecraft were close to Earth in early February 2007. The bottom row illustrates the multiperspective views as the STEREO spacecraft became significantly separated from each other and SOHO later in the year. (The rightmost 

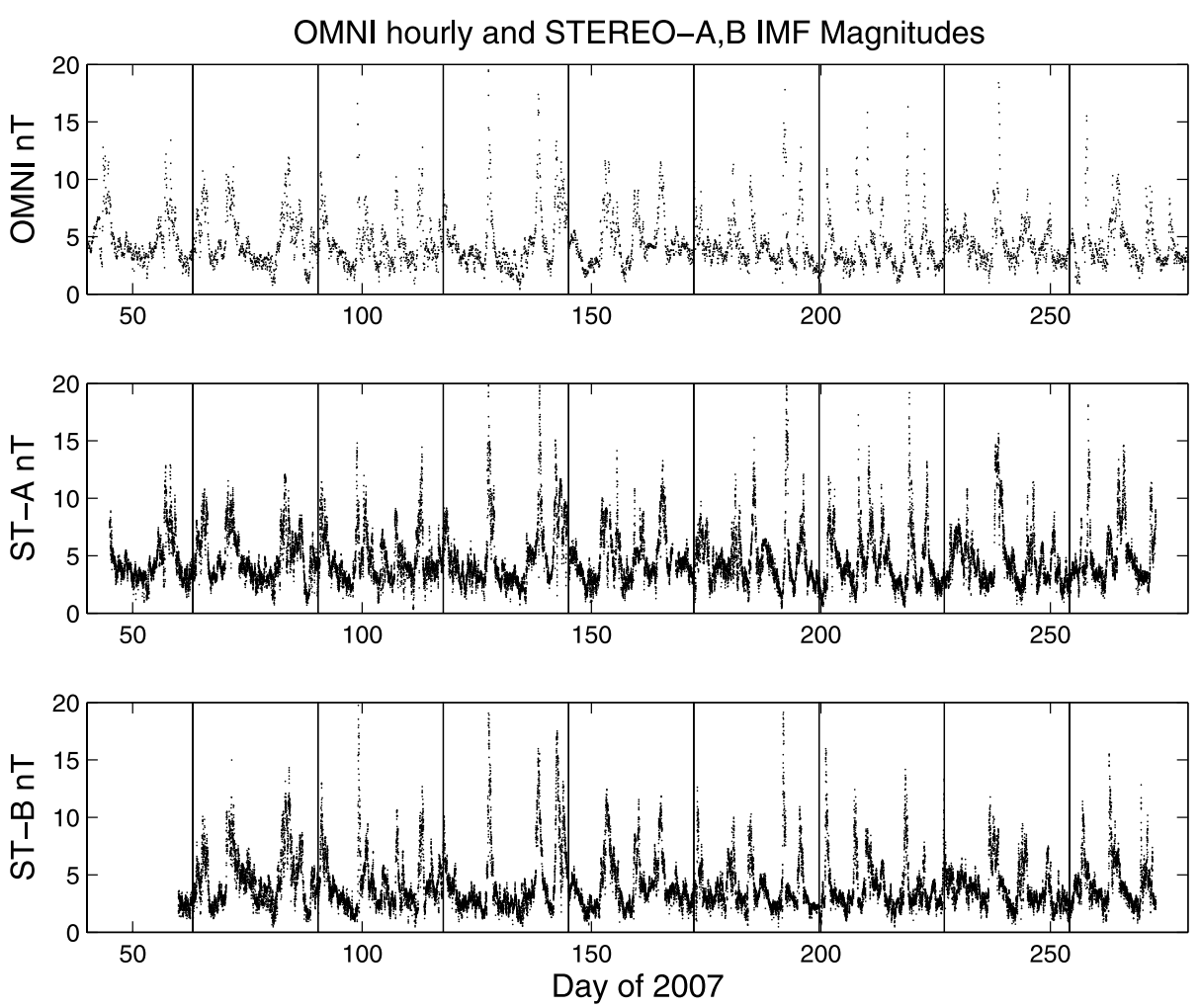

(a)

Figure 2 (a) Magnetic field magnitudes measured at L1 (OMNI hourly data) (top) and on STEREO-A and -B. The vertical lines mark Carrington Rotation boundaries.

panels in Figure 3 are described below.) The solar wind sources in these images are the dark regions on the disks corresponding to open field regions or coronal holes. For a simple dipolar field corona, two polar coronal holes provide the entire heliosphere with solar wind $(e . g$. Pneuman and Kopp, 1971). However, the presence of active regions at low to midlatitudes introduces higher order moments of the coronal field, and with these come a more complex distribution of open field regions that changes as the solar cycle progresses (e.g. Luhmann et al., 2002). The Earth's solar wind on average maps to source regions about $45-60^{\circ}$ to the right (West in the solar imaging convention) of the central meridian, but the source latitude can vary greatly and include low latitude open field regions at some heliolongitudes, polar coronal hole extensions at others, and the edges of the polar coronal holes at yet other locations as the Sun rotates.

For the early months of 2007 two nearly diametrically opposed southern hemisphere coronal holes dominated the solar disk. The EUV synoptic map in Figure 4(a), created by the EUVI investigation team from STEREO-A EUVI images for Carrington Rotation (CR) 2052, shows these sources of the strong, fast solar wind streams seen in the first three Carrington Rotations in Figure 2(c). By late 2007, a large southern polar coronal hole extension, with patches of bright (magnetically closed) field arcades within it (Figure 4(b)), provided a recurrent, long-lived source of more variable high speed wind. A concentrated source region rooted around a low latitude old cycle active region near Carrington longitude $250^{\circ}$ produced 

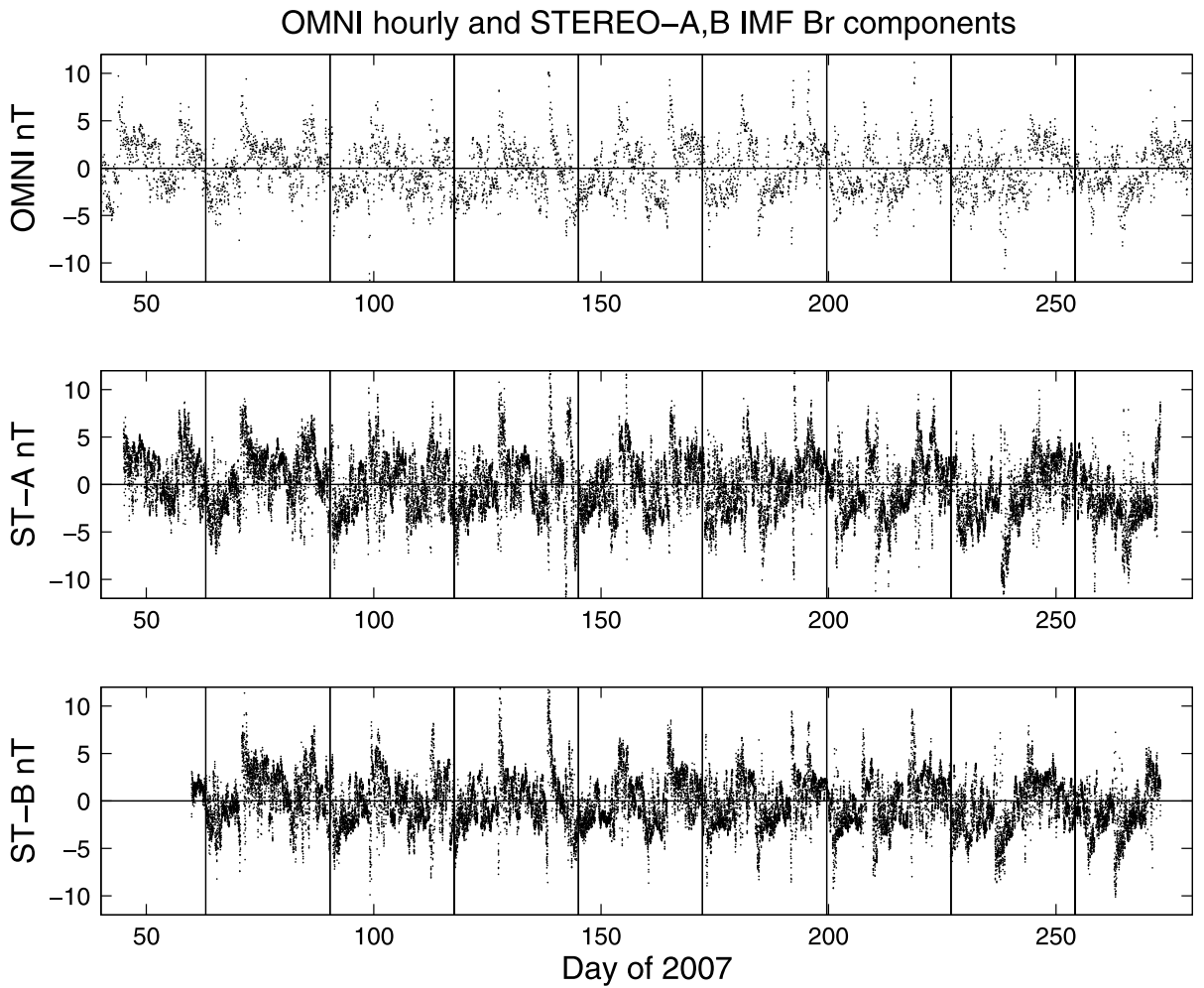

(b)

Figure 2 (Continued.) (b) Comparisons of magnetic field radial components measured at L1 (OMNI hourly data) (top) and on STEREO-A and -B.

a smaller, slightly slower preceding high speed stream. In the following section we use models, such as those illustrated on the right side of Figure 3, to analyze these sources and their implications.

\section{Source Mapping with Models}

A relatively simple description of the coronal topology is provided by Potential Field Source Surface (PFSS) models of the coronal magnetic field (e.g. see the review by Schatten, 1999). PFSS models provide a snapshot of the open coronal field regions, the generally presumed sources of fast solar wind, based on a synoptic map of the observed photospheric magnetic field and the assumption that the coronal field is current free between the photospheric inner boundary and a spherical outer boundary or source surface. The radius of the source surface, together with the multipolar field content of the photospheric boundary map, determine where the model's open fields occur, making the construction of an accurate map from full disk magnetograms a critical part of its successful use (e.g. see Arge and Pizzo, 2000). Investigations using the PFSS model find the best source surface location for describing most observations is located at $\sim 2.5$ solar radii, although values between $\sim 1.6$ and 3.25 solar radii are sometimes adopted. While the current-free and spherical source surface assumptions can be challenged on a physical basis, especially in the vicinity of the he- 

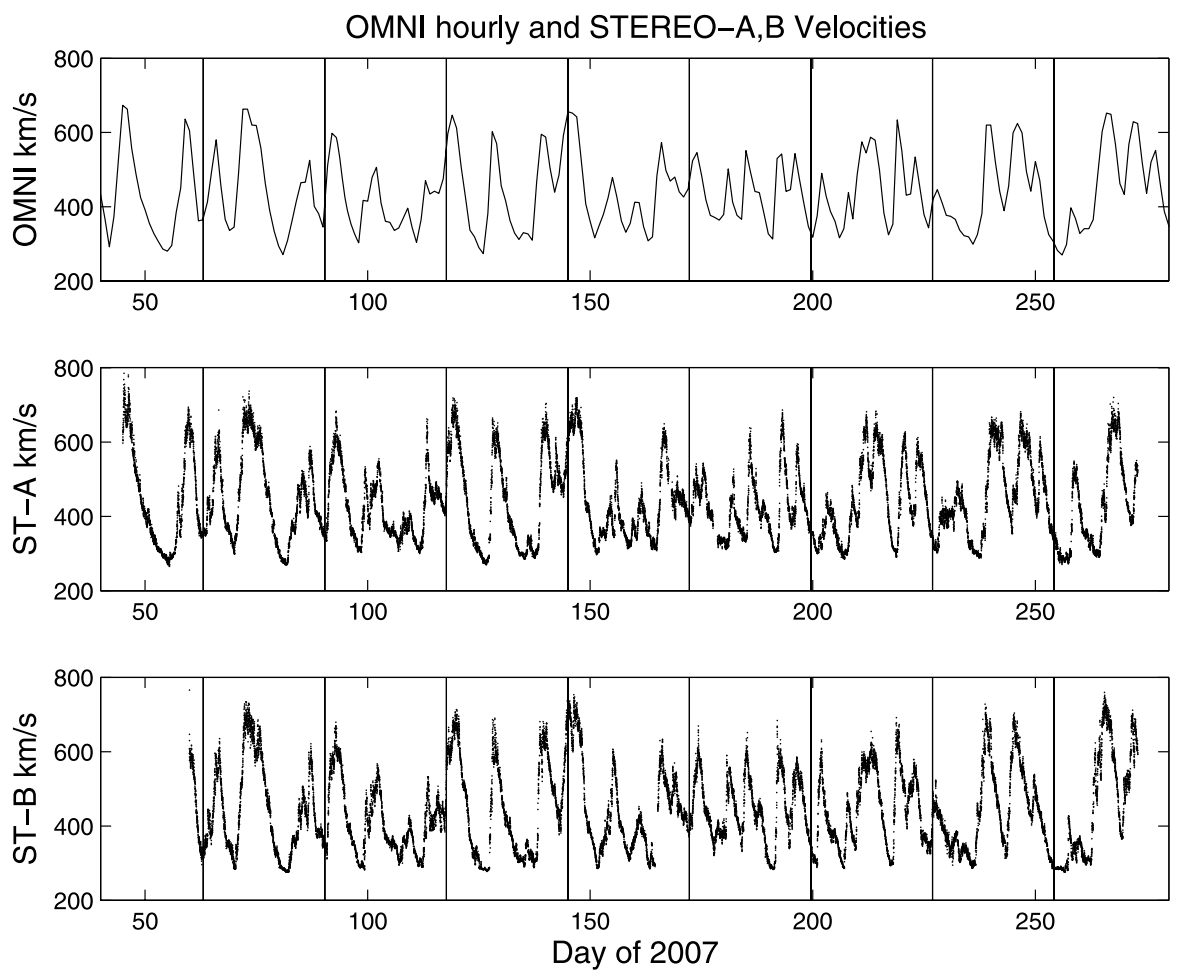

(c)

Figure 2 (Continued.) (c) Comparisons of speeds measured at L1 (OMNI hourly data) (top) and on STEREO-A and -B.
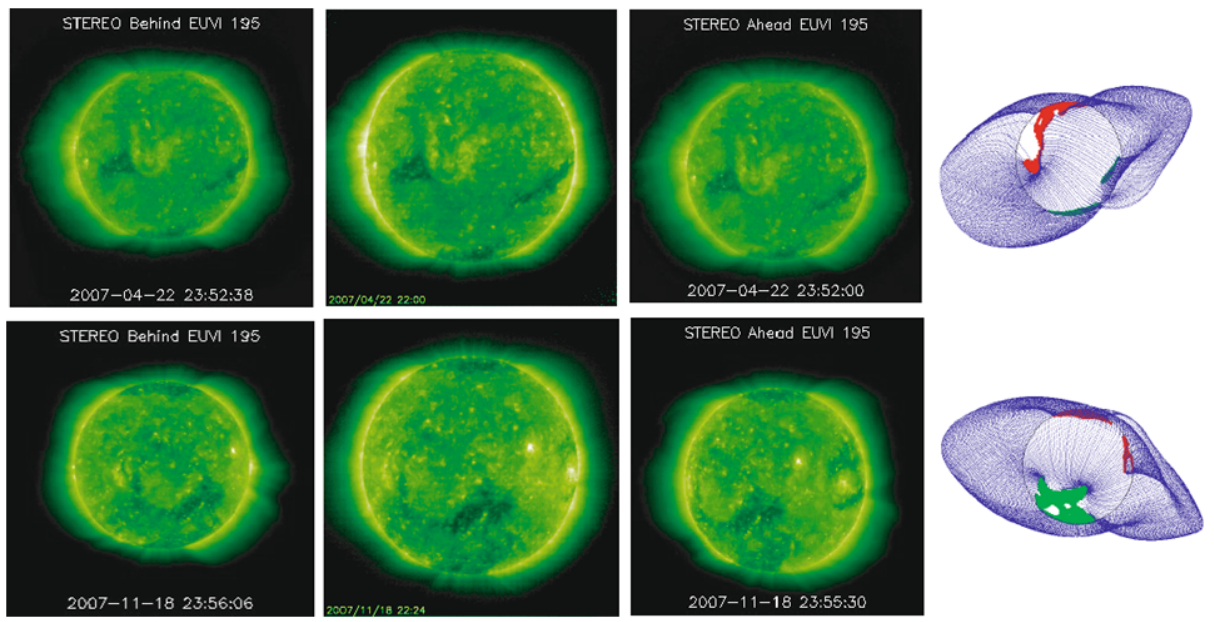

Figure 3 EUV images (left to right: STEREO-B EUVI, SOHO EIT, STEREO-A EUVI) from early (top row) and late (bottom row) 2007. The sources of high speed wind in the ecliptic change from the two southern midlatitude coronal holes in early 2007 to the large southern polar hole extension and a small open field region around a near-equatorial active region (seen near west limb) in late 2007. GONG magnetogram-based PFSS model views from Earth are at the far right, showing areas of outward (red) and inward (green) open coronal magnetic field and the closed field lines at the edge of the streamer belt (blue). 


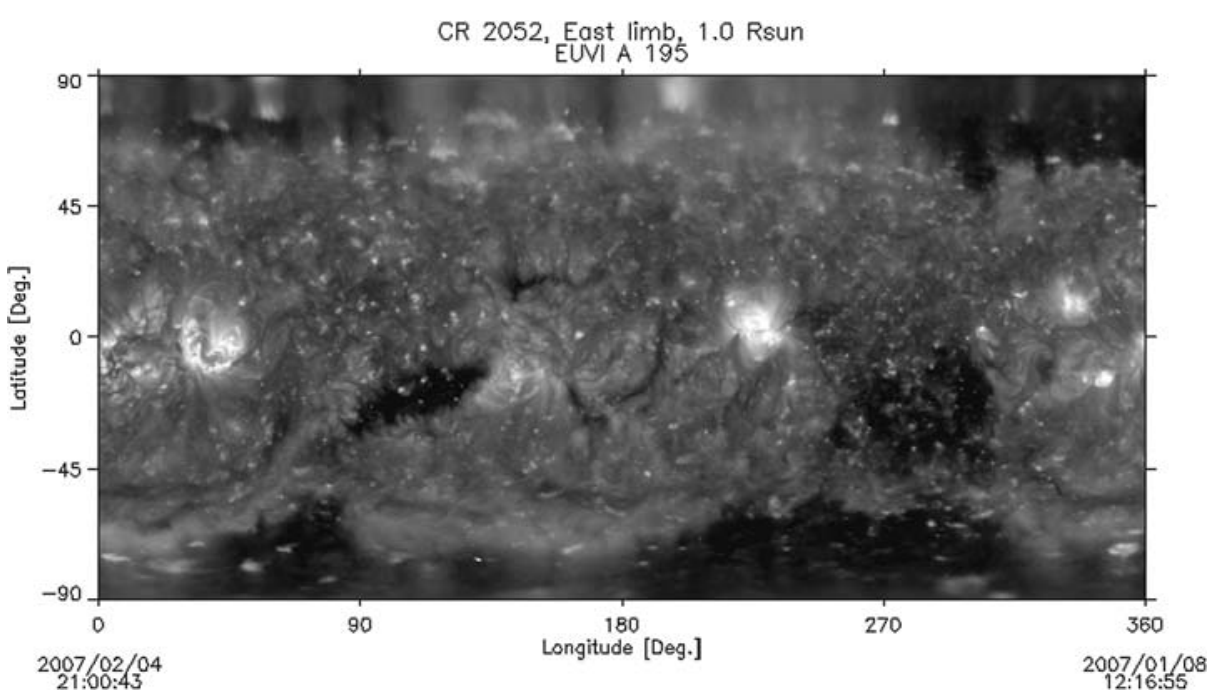

(a)

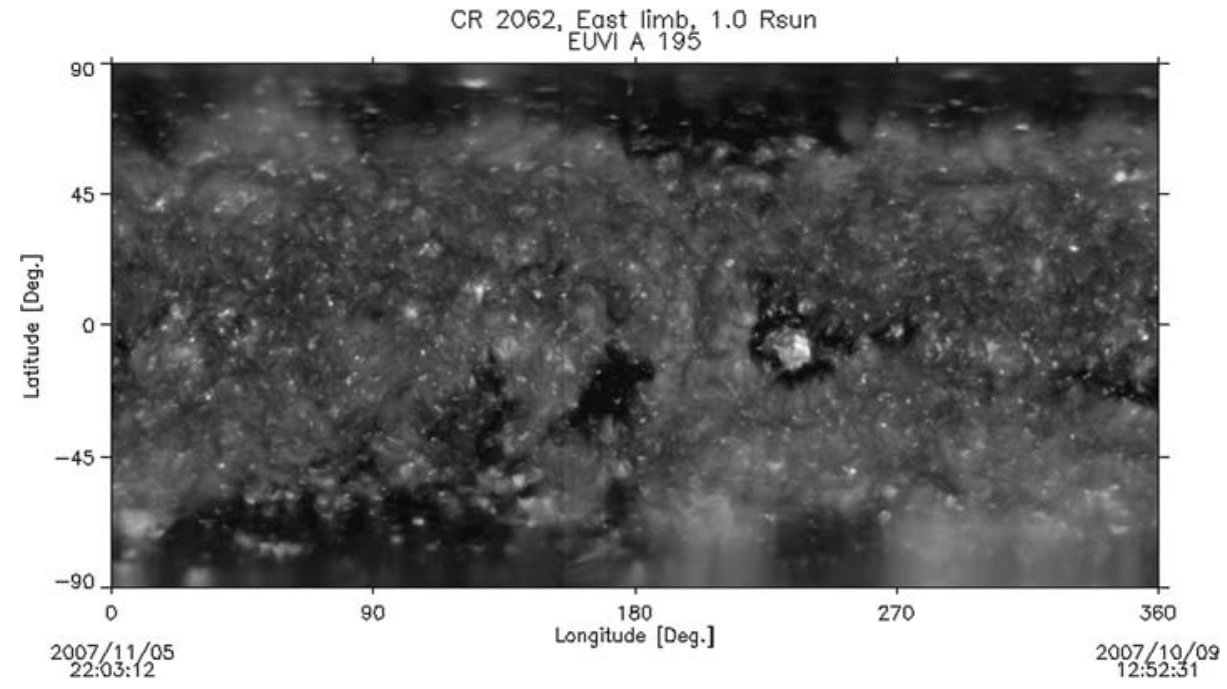

(b)

Figure 4 Synoptic maps constructed from STEREO-A SECCHI EUVI images at $195 \AA$ 年avelength, showing the dark areas (coronal holes) providing solar wind during (a - upper) CR 2052 and (b - lower) CR 2062. Both low-midlatitude and polar holes are present.

liospheric current sheet and around active regions, PFSS models have stood the test of time and applications to many problems requiring realistic large scale coronal field descriptions. In particular, they have been shown to describe many of the gross morphological features of coronal rays in white light eclipse and coronagraph images and the dark coronal holes in X-ray and EUV images, and to reproduce the observed sector structure or polarity of the heliospheric magnetic field when mapped outward (Hoeksema, Wilcox, and Scherrer, 1983; Hoeksema and Scherrer, 1984). One expects the period around solar minimum to be a partic- 
ularly good cycle phase to apply the PFSS model, although a nearly flat heliospheric current sheet may occur at that time, making detailed ecliptic plane comparisons especially sensitive to field boundary conditions or mapping errors.

Examples of the photospheric footpoints of open field regions derived from PFSS models corresponding to the EUV images in Figure 3 are shown at the far right. These particular models are based on GONG magnetograms from the National Solar Observatory, but other magnetic field maps produce the same basic results. In contrast to the traditional PFSS models derived from full Carrington Rotation synoptic maps, the models shown here are updated once or more a day by merging the latest full disk magnetogram into the synoptic map (e.g. Arge and Pizzo, 2000 discuss this process). The model results give insight into the coronal context of the observed open magnetic field regions, their magnetic polarity (red and green areas for outward and inward fields, respectively), and on the largest scale closed field arcade that identifies the helmet streamer belt (blue field lines) - the source of the coronal rays and the heliospheric current sheet base. They also suggest that the coronal field, though largely free of significant active regions, was more complex than a simple dipole, or even a tilted dipole, the 'typical' solar minimum coronal field. The topology of the helmet streamer in early 2007 is affected by a substantial quadrupolar contribution to the solar magnetic field. Even when a more dipolar helmet streamer belt appears later in 2007, there are other closed field regions (so-called pseudostreamers (Wang, Sheeley, and Rich, 2007) rooted in the white areas outside the helmet streamer belt) that complicate the coronal open/closed field boundaries at mid-high latitudes.

The WSA model (Arge and Pizzo, 2000) determines near-ecliptic solar wind sources by extrapolating open PFSS model fields into the ecliptic plane with the aid of a Schatten current sheet model (see Schatten, 1999) in the region between the source surface and $\sim 20$ solar radii $(\mathrm{Rs})$. A modified version of an empirical formula developed by Wang and Sheeley (1990a) is applied to create a description of initial solar wind velocities at 20 Rs based on the divergence of the modeled open coronal magnetic field between the source surface and photosphere. The model velocities are then extrapolated to the ecliptic at 1 AU via a modified kinematic approach described by Arge and Pizzo (2000) that takes stream interactions into account. This empirical model has been further tuned by comparing it to several solar cycles of measured solar wind velocities and interplanetary field polarities, its two outputs. Considering its semi-empirical construction based on the statistics of speeds in earlier cycles, and its simplified physics and assumptions, this model sometimes does a remarkable job of reproducing the time series of velocities during periods of undisturbed or even moderately disturbed solar wind (Arge and Pizzo, 2000; Lee et al., 2008).

Source mapping to the ecliptic using the WSA model based on Mt. Wilson Observatory magnetic field maps are shown in Figure 5(a) and (b) for both the early and later 2007 examples in our time period of interest, respectively. Here full Carrington Rotations are shown but updating synoptic maps produce the same basic pictures. In early 2007, the model suggests that the prominent midlatitude southern hemisphere open fields (referred to here as midlatitude coronal holes) seen in the corresponding EUV images in Figure 4(a) occurred in deep quadrupolar warps of the helmet streamer belt. A four-sector interplanetary field pattern results from this geometry, with the two southern hemisphere midlatitude holes feeding the low latitude solar wind with its (positive) polarity sectors and the (negative) polarity portion coming from a few weak northern hemisphere polar coronal hole extensions. The four-sector magnetic polarity pattern is evident in the observed radial field component in the left portion of Figure 2(b). As previously mentioned, in later 2007 the main sources of solar wind changed to a large southern polar coronal hole extension and a smaller low latitude 


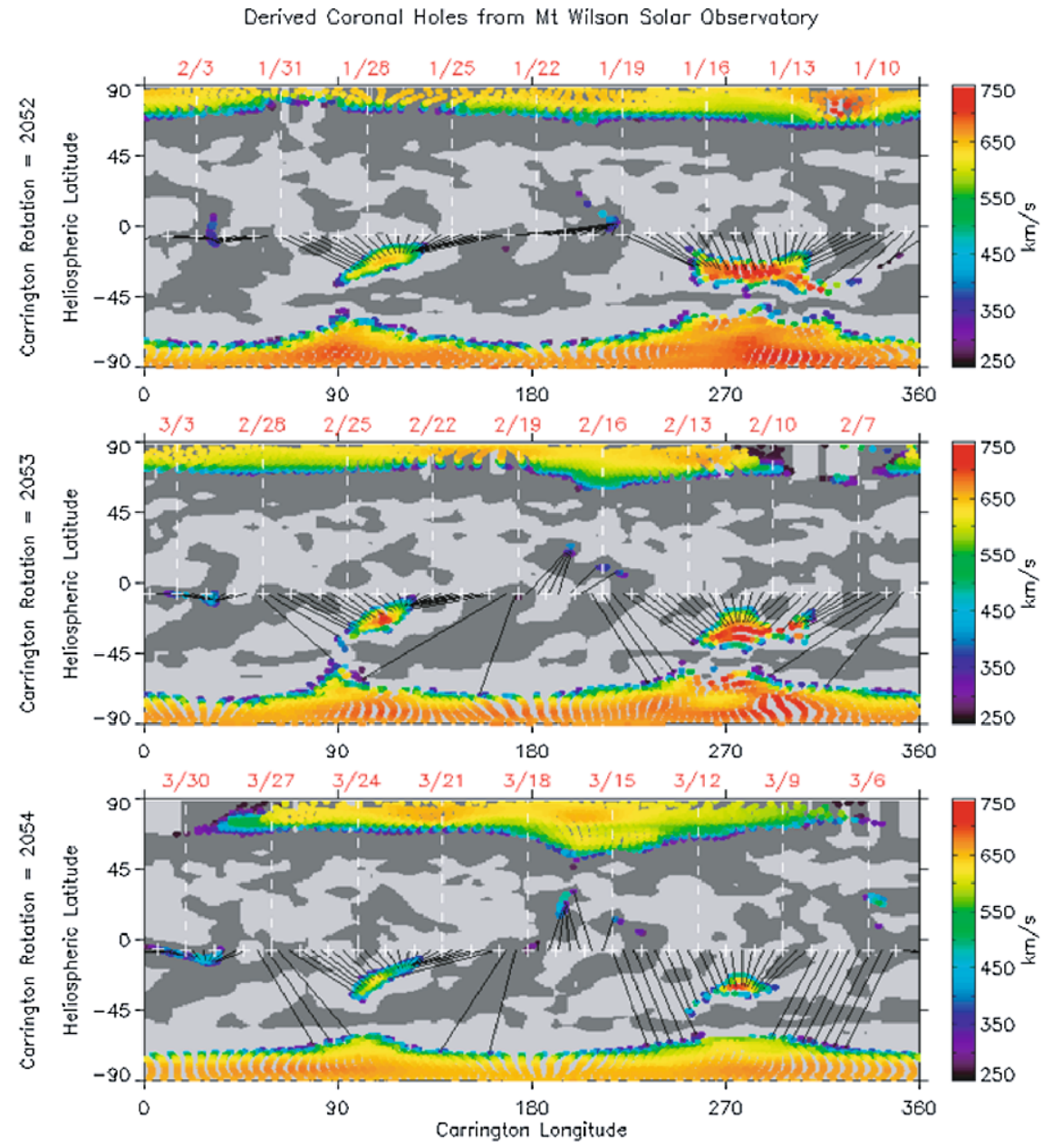

Created 2007 Apr 92207 UTC

NOAA/SEC, BOULDER, CO, USA

(a)

Figure 5 Early 2007 (a - above) and late 2007 (b - next page) ecliptic solar wind source mappings from the NOAA SWPC WSA model, showing the increased contribution of the polar coronal hole edges in the latter period. (From the NOAA SWPC website http://www.swpc.noaa.gov/ws/.) The model open field region footpoints on the photosphere are color coded according to the model solar wind velocity that flows from those locations (colorbars at right of maps). The light and dark gray backgrounds indicate the magnetic polarity of the photospheric field on which the models are based.

hole that was located in the vicinity of an old cycle active region. However, in this case the corresponding model in Figure 5(b) (whose open fields agree with EUV images in Figure 4(b)) suggests that for about half the solar rotation, the ecliptic solar wind comes from the edges of the polar coronal hole, the more traditional solar minimum source region.

Figures $6(\mathrm{a}, \mathrm{b})$ compare the WSA model velocities and interplanetary field polarities with the OMNI in situ solar wind data from the early and late 2007 periods. In this case daily updated maps based on Mt. Wilson Observatory magnetograms were used for the 


\section{Derived Coronal Holes from Mt Wilson Solar Observatory}
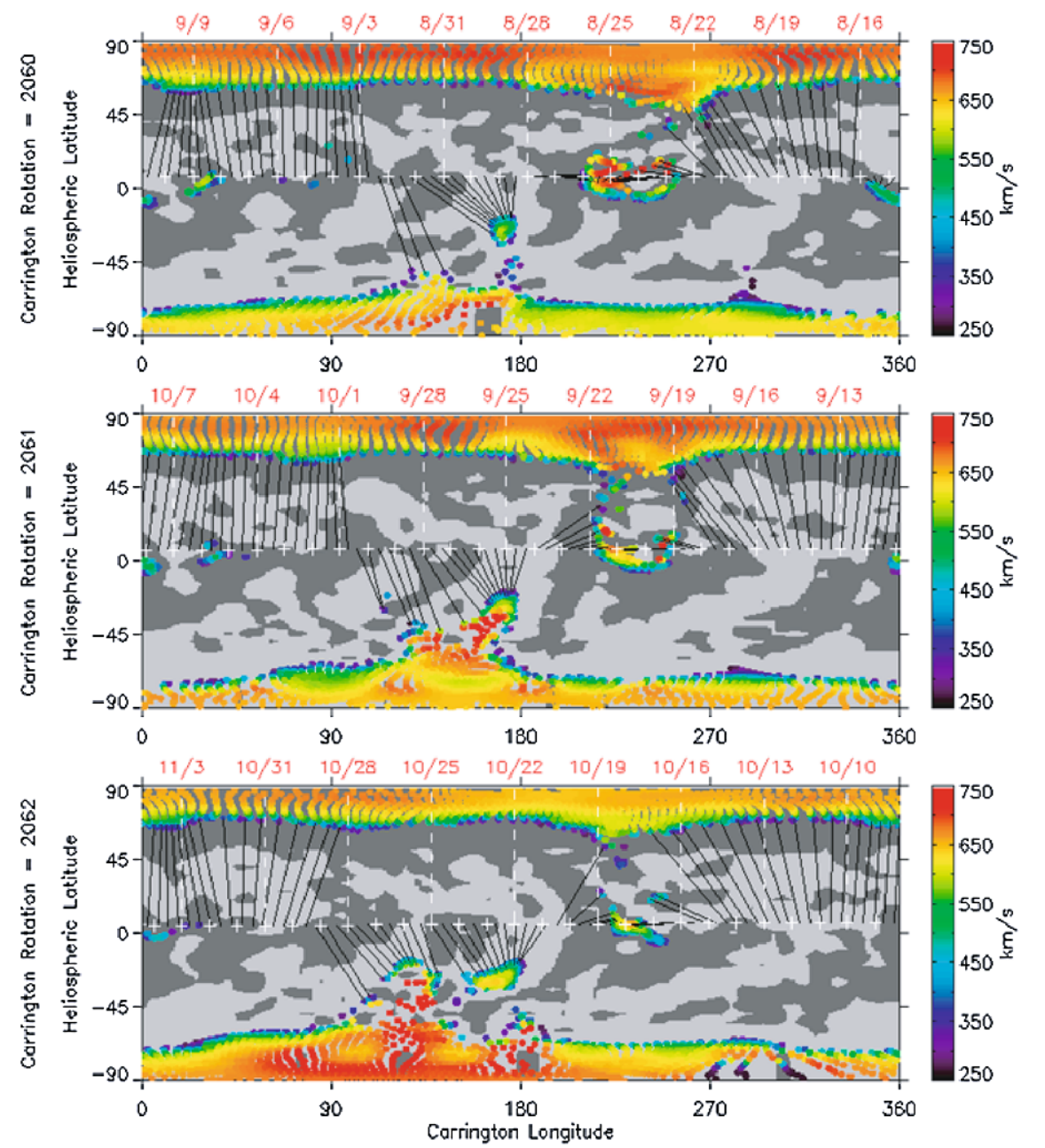

Created 2007 Nov 132107 UTC

NOAA/SWPC, BOULDER, CO, USA

(b)

Figure 5 (Continued.)

WSA model PFSS boundary condition. Considerable differences in model agreement can be seen by inspection of the time series for the two periods. The early 2007 model generally reproduces the major high speed streams and field polarities in the measurements, but the later 2007 period fails to capture the details of the observed stream structure. Moreover, the model field polarities in the latter case exhibit fewer sector boundary crossings than the data. The reasons can be related to the source mapping results in Figure 5(a, b). It was noted above that in early 2007 the ecliptic plane maps mainly to the southern midlatitude coronal holes present at the time, while in late 2007 it maps at least half the time to the polar 

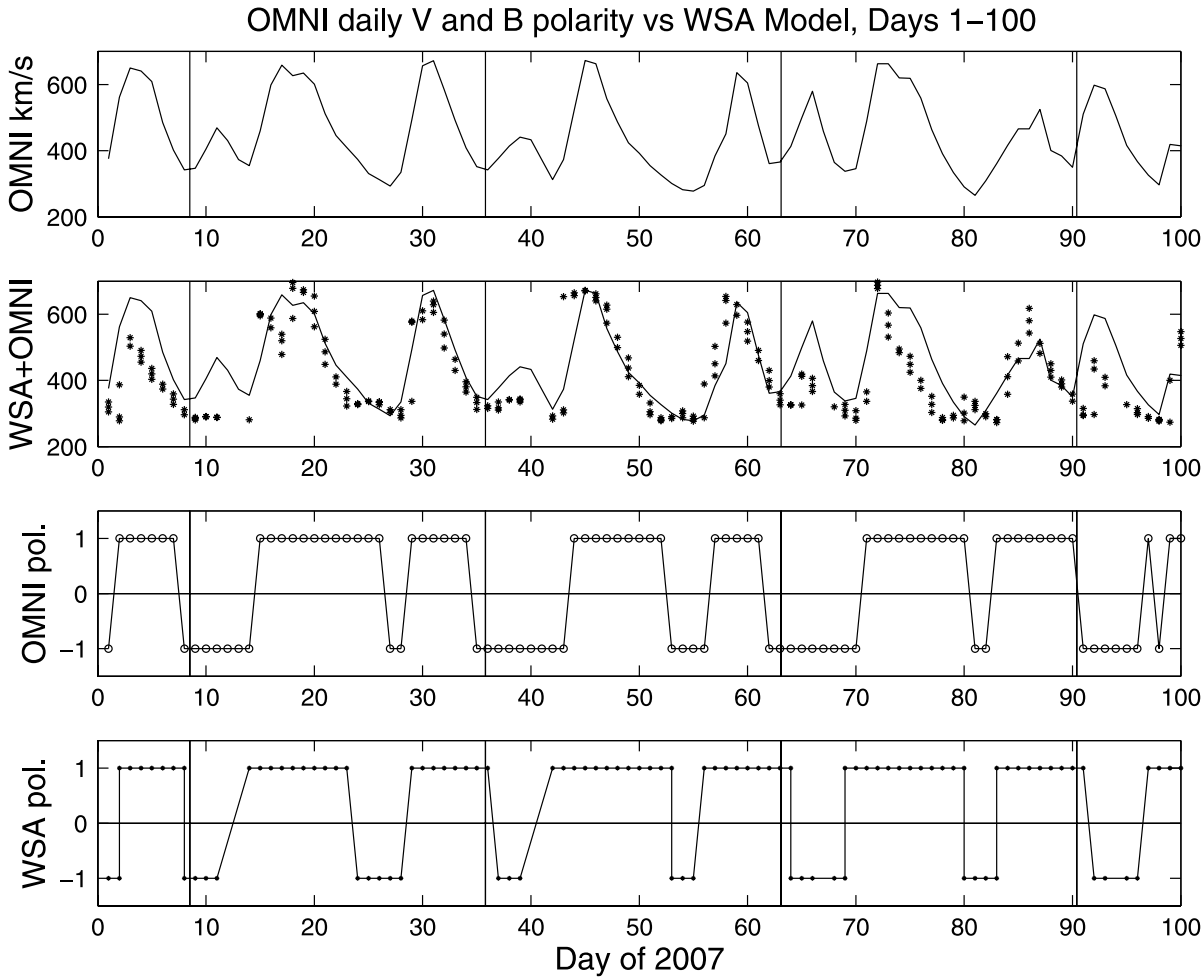

(a)

Figure 6 OMNI solar wind speeds (top panels), calculated WSA model speeds (asterisks) overlaid on the OMNI data (second from top panels), OMNI and WSA model interplanetary field polarities (second panels from bottom and bottom panels, respectively) for the early (a - above) and late ( $b$ - next page) 2007 periods of OMNI L1 data in Figure 2. Field polarities are +1 for outward pointing fields and -1 for inward pointing fields.

coronal hole edges. The PFSS model framework can provide insight into how the coronal field determines which sources prevail.

As discussed by Arge and Pizzo (2000) and others, polar field corrections to synoptic maps constructed from full-disk magnetograms are necessary due to the poor (and changing) visibility of the Sun's polar regions from Earth. The PFSS models show us that the areas and shapes of the open field regions are sensitive to the combination of the polar field strength, the active region field strengths and distribution, and the effective source surface radius. If the source surface radius is not significantly varying, e.g. due to an overall change in the heating and/or energetics of the corona, and the active region field contributions are minimal or fixed, then the polar fields have controlling influence on the open fields. The relative sizes and shapes of the low latitude open field regions and the polar open field regions can be altered in the model by introducing a different polar field description. In particular, the ecliptic source mappings that are border-line between midlatitude and polar coronal hole edge connections are especially sensitive to such changes. This could explain the relative disagreement of the observed and model stream structure in late 2007 seen in Figure 6(b). Ecliptic mapping to a coronal hole edge would generally produce a low model velocity, while mapping to a more centrally located part of a midlatitude coronal hole or 
OMNI daily V and B polarity vs WSA Model, Days 175-275
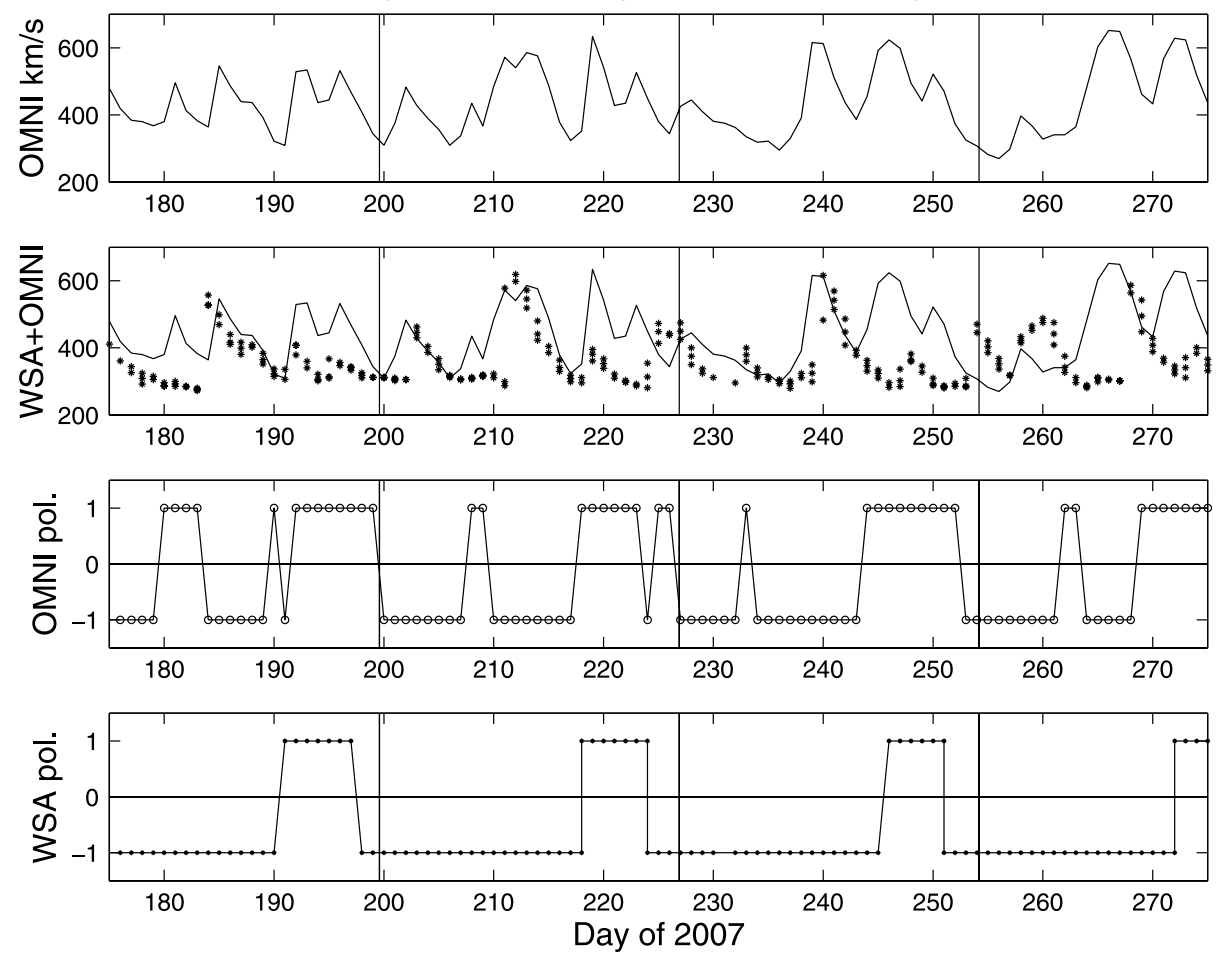

(b)

Figure 6 (Continued.)

open field region could produce a relatively high model velocity. Such sensitivity to the polar field distribution may explain the observed solar wind and the difficulty in modeling the late 2007 period.

\section{Influence of This Cycle's Solar Polar Field}

It has already been pointed out in the literature (e.g. Smith and Balogh, 2008) that this cycle's photospheric field is characterized by polar fields $2-3$ times weaker than at this phase of the previous two cycles (also see Lee et al., 2009). McComas et al. (2008) analyzed the solar wind mass flux seen on the most recent Ulysses polar pass and found it to be less than the previous solar minimum pass mass flux by about $20 \%$. Most of this decrease appears to be associated with lower densities rather than lower speeds. The authors also found similar changes in both L1 data and at low heliolatitudes, indicating a global rather than high latitude effect. These observations have increased interest in the role of the solar polar magnetic field on the structure of the heliosphere, as well as its implications for solar wind and interplanetary field characteristics.

Figure 7(a) shows annual statistics of the measured solar wind speeds from the OMNI data set for the four years including the late declining and minimum phases of the last two cycles and for the recent cycle through most of 2007. The comparison of 2007 OMNI with 


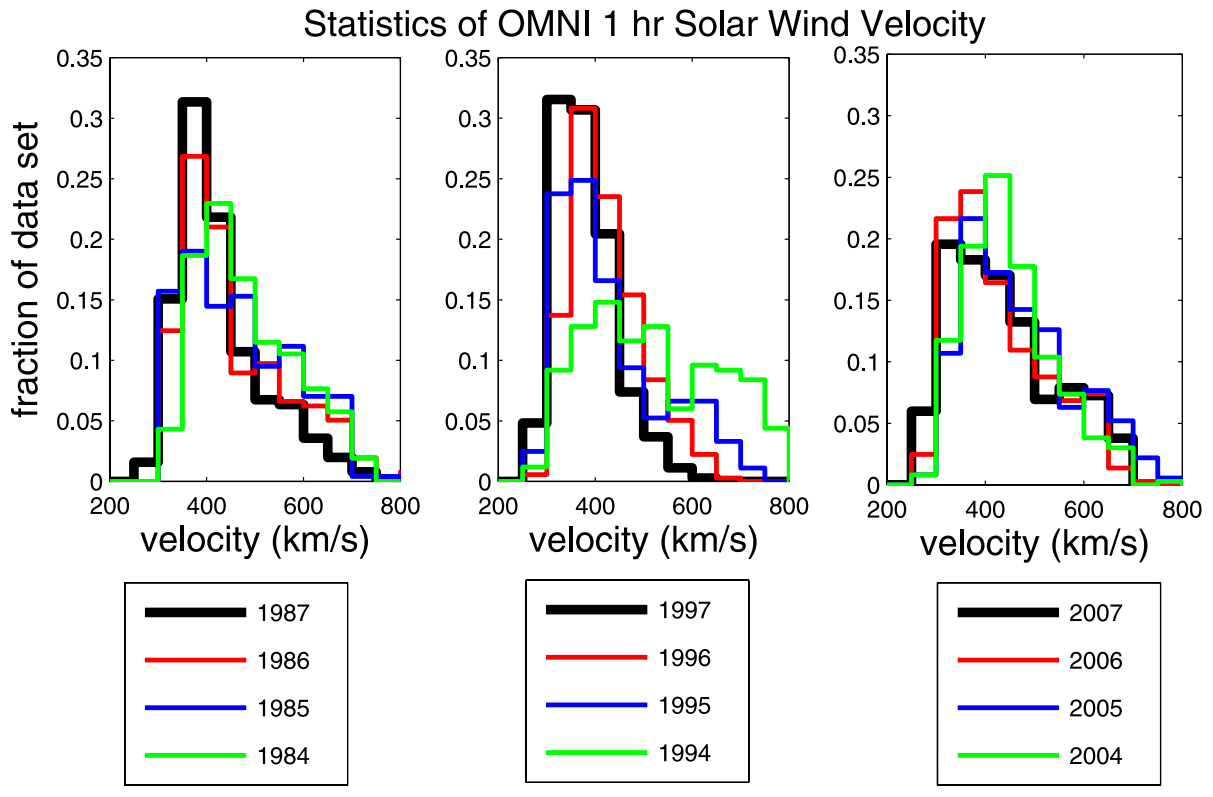

(a)

Figure 7 (a) The observed annual solar wind speed distributions for the four years surrounding the last two cycle declining and minimum phases (left and center panels), and the recent cycle longer declining phase (right panel). The histograms in each panel are colored in a sequence from green to blue to red to black as time progresses through each late declining phase and minimum. Compared to the two previous cycles, the current cycle (right) has consistently exhibited speed distributions with lower occurrence of slow wind and pronounced high speed tails, characteristic of the early years shown for the previous cycles. Note the greater dominance of low speeds and reduction of high speeds that occur as the cycle ramps up from the minima in 1987 and 1997, features not yet occurring in 2007 (black histograms in all panels).

STEREO-A and -B data in Figure 7(b) demonstrates that the recent speed distribution is not unique to the $\mathrm{L} 1$ perspective or to the instrumentation and data processing method. The latest period (right panel, Figure 7(a)) has nearly four years of consistently flat distributions with high speed tails while the two previous cycles had only about two such years. This difference could be simply due to the longer duration of the present cycle declining phase. Indeed, the speed distributions from the early years of the two previous cycle periods, isolated in Figure 7(c), have characteristics similar to the latest cases. However, as will be suggested below, the weak polar fields of this cycle may also contribute.

Luhmann et al. (2002) used the PFSS model to visualize the evolution of solar wind sources over $\sim 2.5$ solar cycles, using Mt. Wilson Observatory archived synoptic maps at the inner model boundary. In Figure 8(a) we extend their plot of the model coronal holes in a time-series constructed by reversing Carrington Rotation maps of the computed coronal holes and stringing them together. Figure 8 (b) shows sunspot number on the same timeline, with starting times for the years marked by vertical lines, including the years analyzed in Figure 7(a) (blue lines). Altogether, this display shows the difference between the (modeled, and from selected image comparisons, also observed) coronal holes for the current declining phase compared to the two previous cycles. The open field regions in the previous two cycle declining phases were dominated by polar holes and their extensions. In contrast, the current cycle declining phase has a persistent mid-low latitude band of open field regions. Figure 9 zooms in on the open fields for the four years around the cycle minima only, adding in 


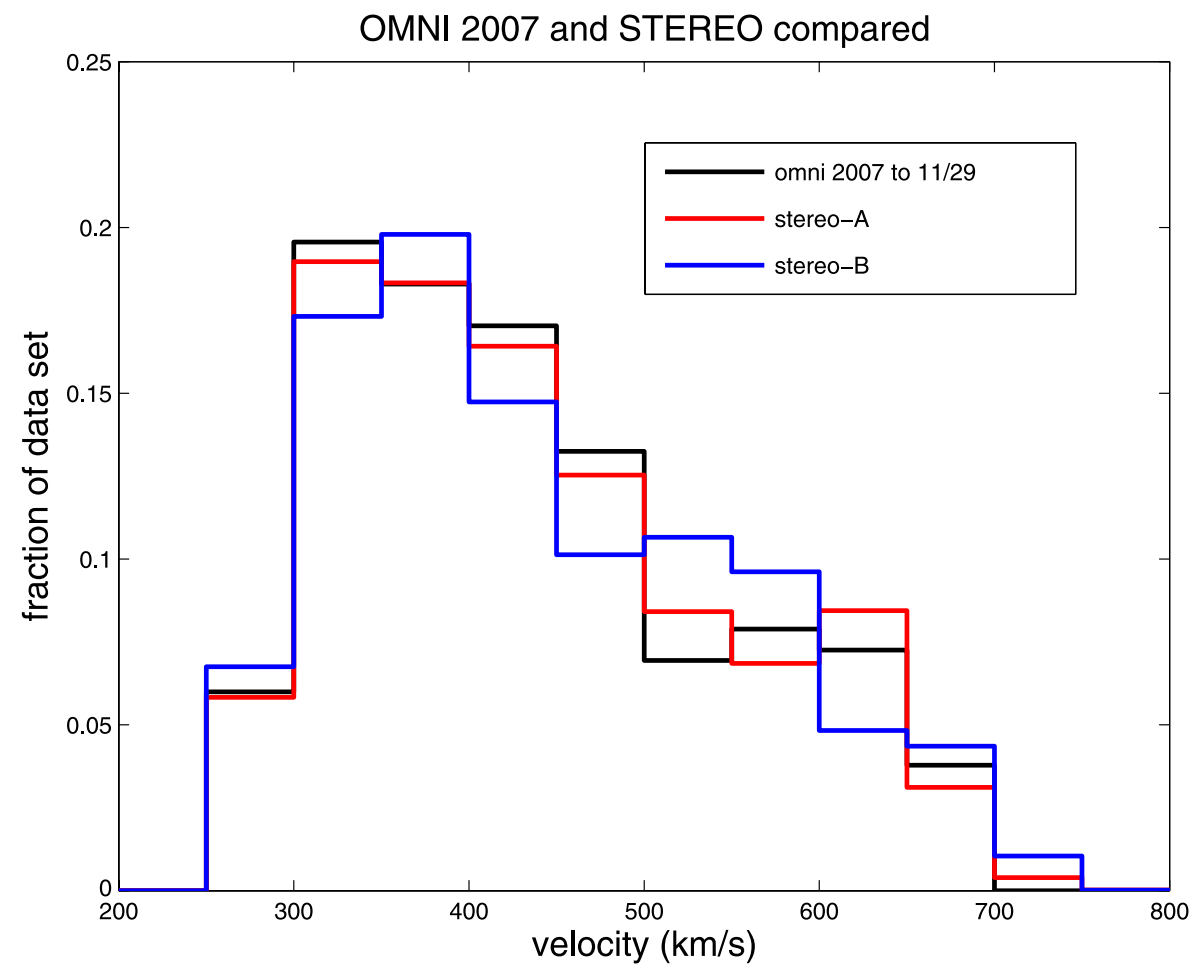

(b)

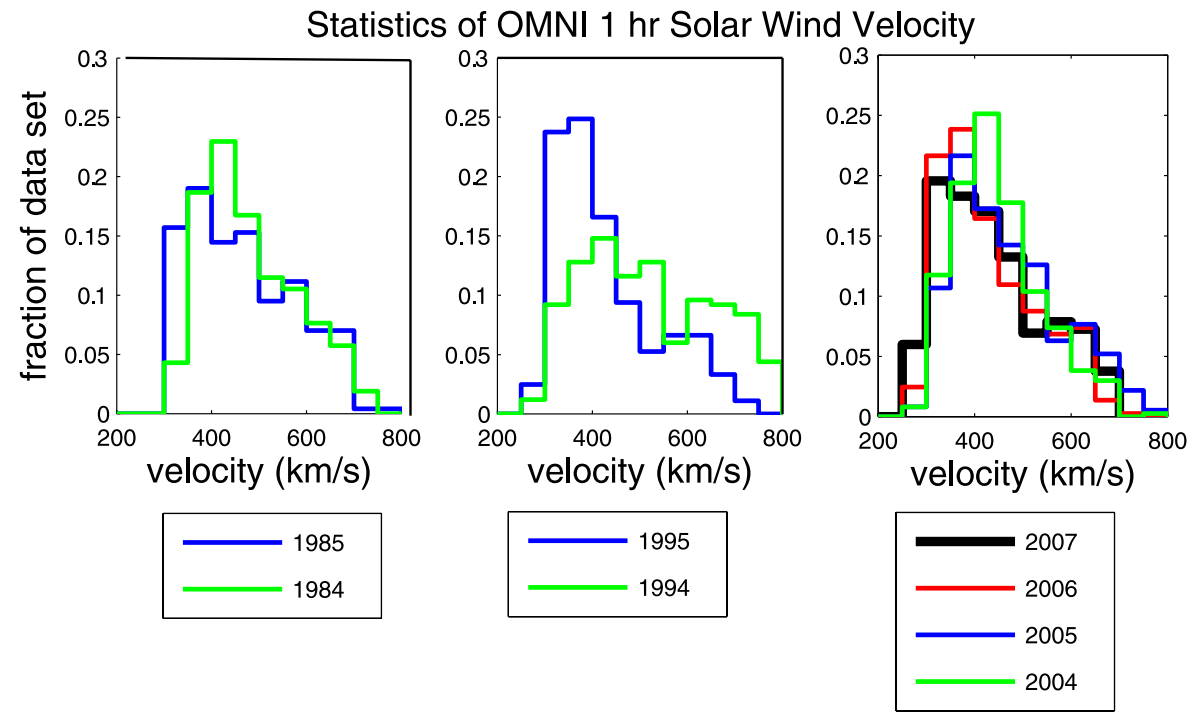

(c)

Figure 7 (Continued.) (b - upper) The similarity of the 2007 OMNI and STEREO-A, -B speed distributions indicates that its characteristics are not unique to the OMNI L1 data or site. (c - lower) Same as (a), but here showing only the annual speed distributions for the late declining phase years of the two previous cycles. This illustrates the similar appearance of these particular speed distributions and those persisting throughout the recent period including the STEREO years. 

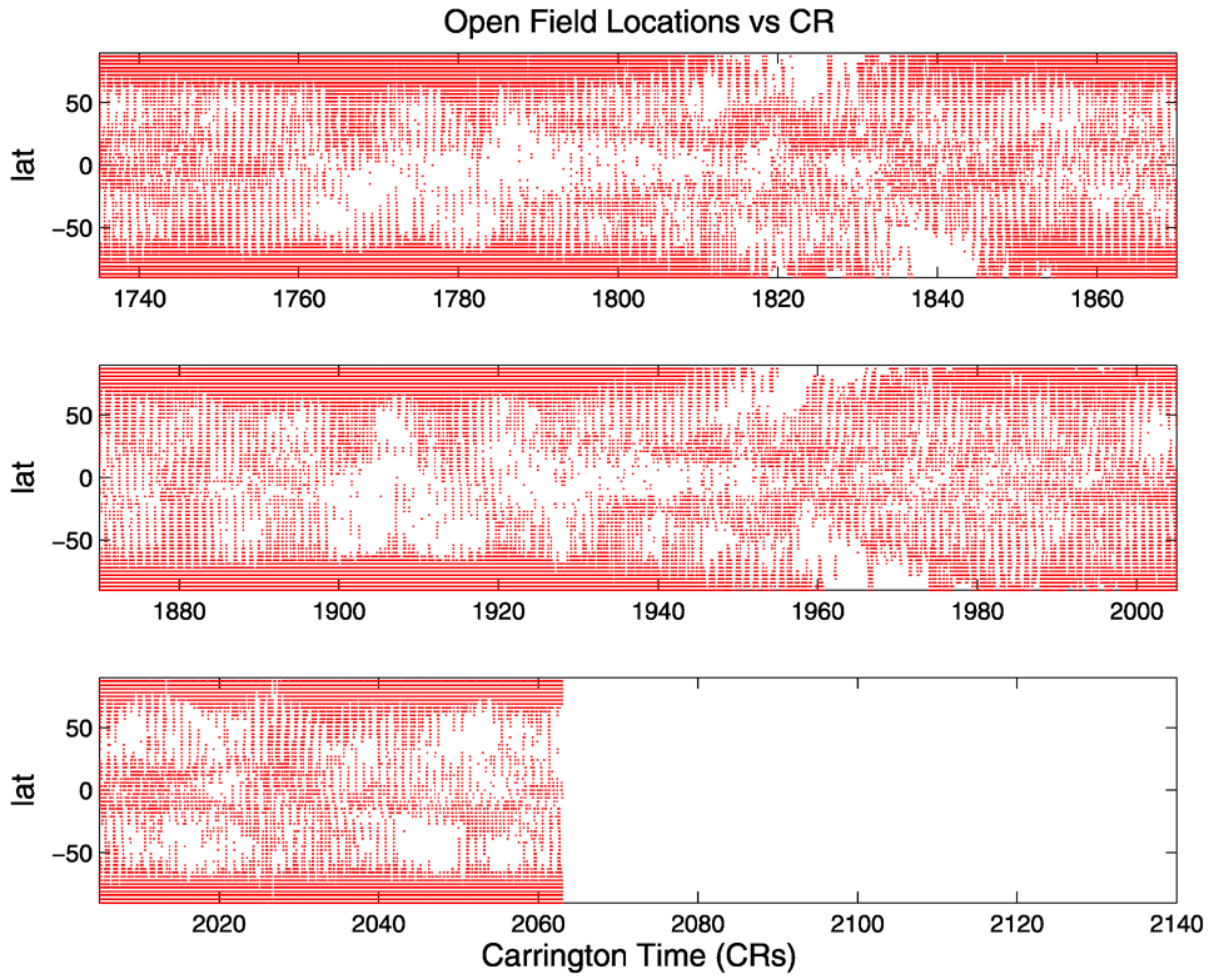

(a)

Figure 8 (a - above) Extension of a plot in Luhmann et al. (2002) of PFSS model open field regions on the photosphere derived from Mt. Wilson Observatory magnetograms. Each panel represents a solar activity cycle, from the late declining phase through the maximum, as illustrated by the sunspot numbers in (b-next page). The dotted areas represent the coronal hole or open field footprints in synoptic map form, laid end to end. This plot can be thought of as representing the coronal hole areas that pass the central meridian as a function of time. Here one can distinguish between high latitude or polar coronal hole regions and their extensions, and low latitude open fields associated with the activity belts at mid to low latitudes. The late declining phase OMNI and STEREO observations discussed in this paper occur during Carrington Rotations 2052 - 2062 (bottom panel).

contrasting colors the specific model footpoints connecting to low ecliptic latitudes. These mappings most closely represent the sources affecting the OMNI and STEREO data. The major contribution of the persistent mid-low latitude coronal hole sources to this cycle's declining phase solar wind distinguishes it from its predecessors. Whether this difference affects the observed speed distributions of this cycle is addressed below.

The reason for this cycle's different polar field is thought to be the slower meridional flows inferred from surface feature tracking (e.g. Hathaway, 2008). Solar dynamo models based on the flux transport concept (e.g. Wang and Sheeley, 1990b) suggest that a net poleward motion of weak solar surface fields seen in observations is part of a hemispheric Hadley-cell like circulation pattern that returns the cycle's emerged magnetic flux to the base of the convection zone. This large-scale motion, coupled with supergranular diffusion of the fields and apparent cancellation of opposing fluxes brought into contact, determines how the observed flux from emerged active regions both decays and is redistributed to form the large scale quiet Sun field. For yet unknown reasons this cycle exhibits a slower poleward migra- 


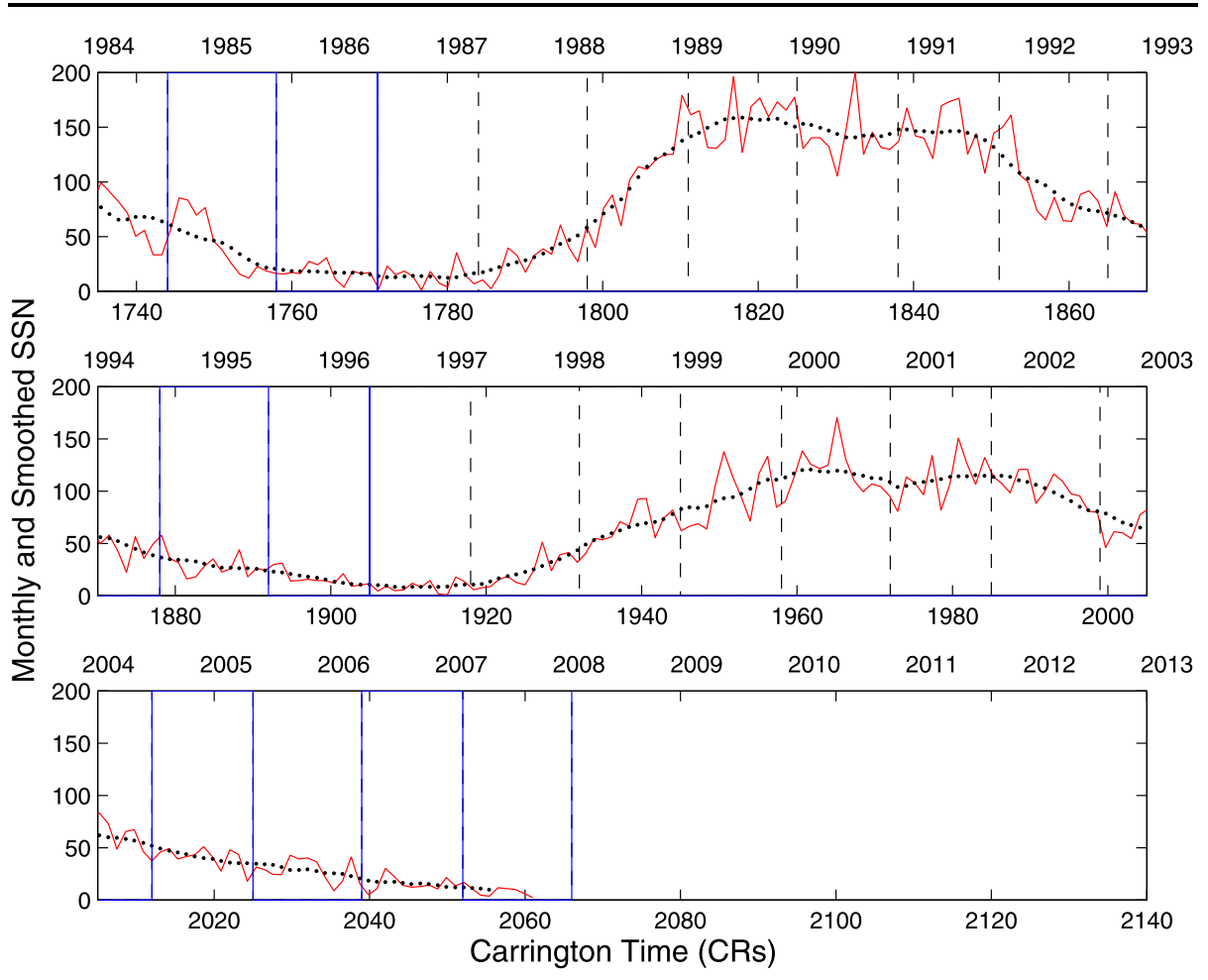

(b)

Figure 8 (Continued.) (b) The red and dotted lines show monthly and smoothed monthly Zurich sunspot numbers corresponding to the panels in (a).

tion of the fields (e.g. Schrijver and Liu, 2008), which thus have more time to diffusively decay as positive and negative fields interact. The result is weaker polar fields that should in turn affect the next solar activity cycle, but they may also be affecting the ecliptic solar wind sources at this time.

An experiment can be done with the PFSS and WSA models to approximate the consequences of a weaker polar field on this cycle's solar wind. Figure 10 shows PFSS model ecliptic field source mapping for Carrington Rotation (CR) 2051, from early 2007, based on Mt. Wilson magnetograms, compared to a similar mapping when the axial dipole contribution to the spherical harmonic coefficients is increased by threefold. The result of the actual (weaker) polar fields (top panel) is a significantly more important contribution of the midlatitude coronal holes and polar hole extension to the mapped ecliptic solar wind than may otherwise have occurred.

In the WSA model, the polar field corrections applied to the synoptic maps are based on the analysis of the full disk magnetograms from the previous two solar cycles (Arge and Pizzo, 2000). To test the above hypothesis, we ran the WSA model for the recent period using specially prepared synoptic maps wherein the polar fields were either reduced by threefold, or increased by threefold. These runs were made by carefully altering the original maps to ensure that the adjustments to the polar fields did not introduce any monopoles or other undesirable behavior. The alterations were restricted to latitudes above $\sim 30^{\circ}$, where they were introduced with a latitude dependence that smoothly increased towards the poles. 

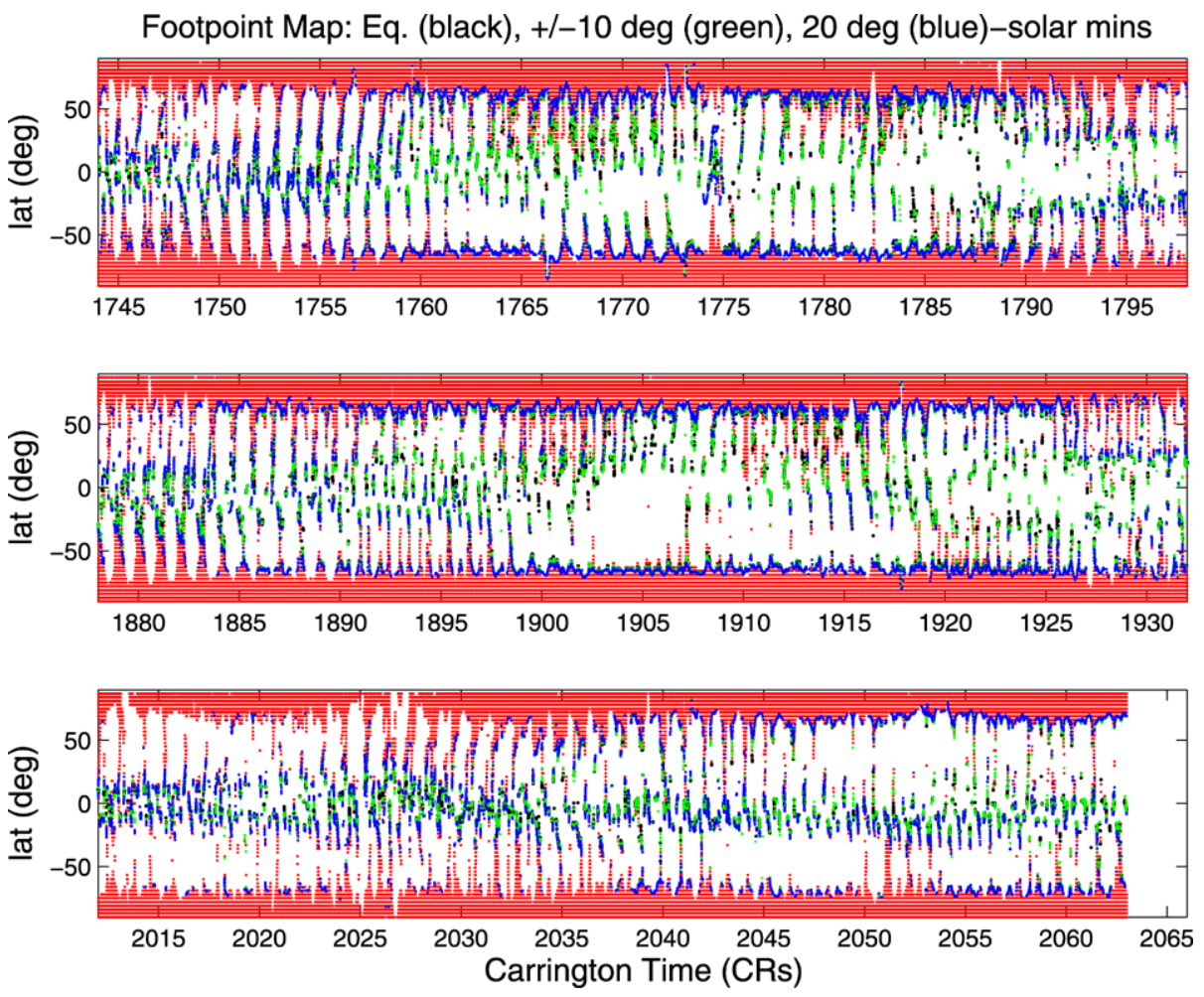

Figure 9 Zoom-in on the late declining/minimum phases in Figure 8(a), with footpoints mapping to low heliolatitudes $\left(0^{\circ}, \pm 10^{\circ}, \pm 20^{\circ}\right)$ identified by the black, green and blue points. The persistent band of low latitude sources in the recent period (bottom panel) is central to the present study of the cycle differences and the effects of the weak solar polar fields.

The revised model speed statistics for the period under study, compared to the original model statistics, are shown in Figure 11. As might have been anticipated from the PFSS model experiment in Figure 10, the speed histogram becomes flatter and develops a more prominent high speed wing in the weakest polar field WSA model for two reasons. First, the weaker polar fields make the low-midlatitude coronal holes larger. Second, the ecliptic more often maps deep inside the midlatitude open field sources. Although they are not a perfect match to the observed speed distributions in Figure 7, the trends in Figure 11 agree with the differences between the previous cycles and the latest cycle. Thus the present cycle declining phase and early minimum Sun may be producing more high speed solar wind than would have occurred had the solar polar field been similar to previous cycles. This is an interesting example of how the solar dynamo can have significant $1 \mathrm{AU}$ effects beyond the occurrence rates of flares and CMEs.

\section{Conclusions}

The new availability of STEREO solar wind measurements for investigating quiet solar wind behavior and testing currently used models of the solar wind prompted the present analyses of the cycle 23 declining phase and early minimum period. The results may have 


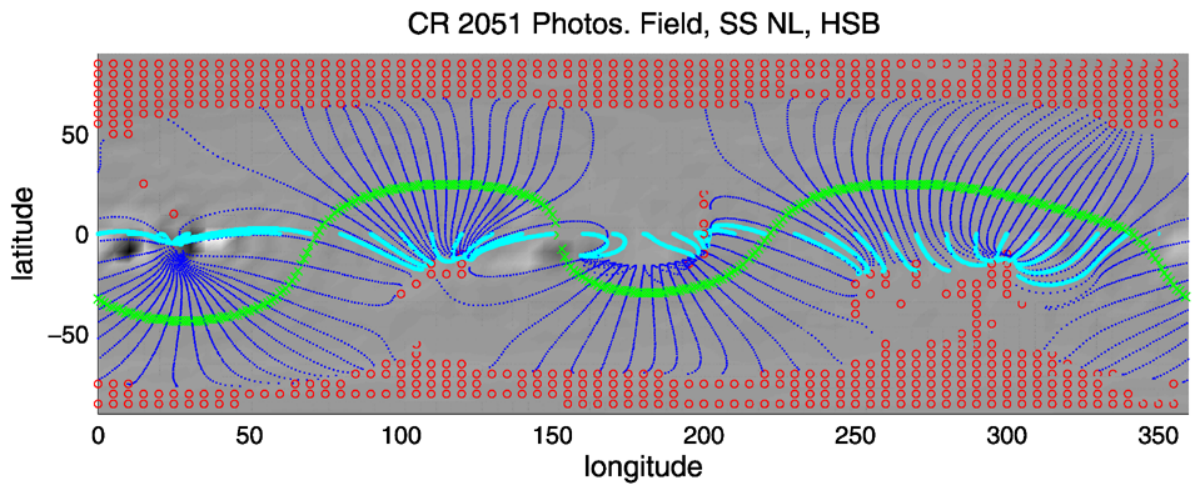

CR 2051 Photos. Field, adjusted polar field

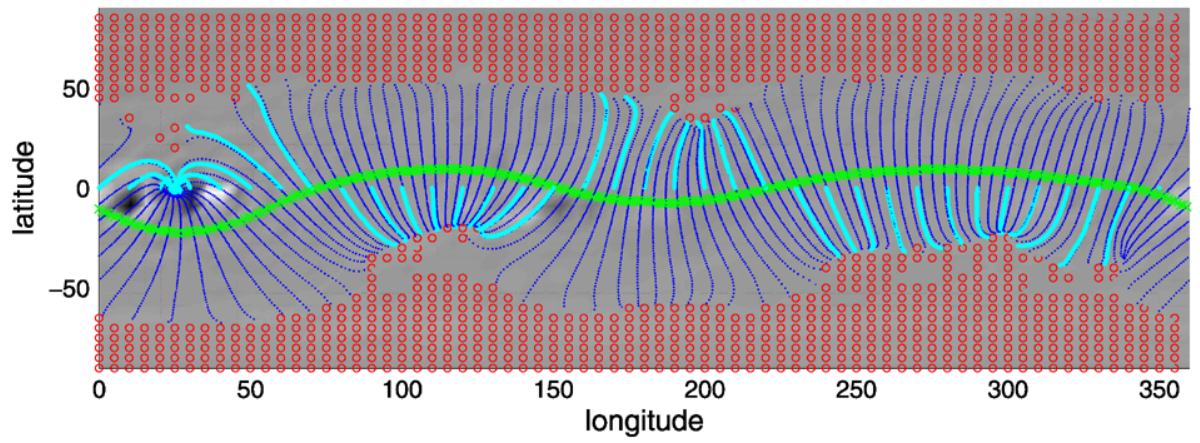

Figure 10 An experiment with an altered polar field in the PFSS model is illustrated by this Carrington Rotation 2051 result. The gray scale shows photospheric fields obtained from solar magnetograms. The red stippling indicates the footpoints of model open field regions (coronal holes), the sources of the solar wind. The dark blue field lines are part of the helmet streamer belt and the green line is the magnetic neutral line on the source surface of the model. The cyan field lines are those that map to the equatorial plane, approximately locating the ecliptic solar wind sources. In the top panel the polar dipolar field contribution is as observed. In the bottom panel it is increased by a factor of three to more closely resemble its magnitude in the two previous solar cycles. A consequence of the weaker polar field is that the source mapping is to the low-mid latitude open field regions rather than to the polar hole edges and extensions. The weak polar field also reduces the polar hole size relative to the mid-low latitude coronal holes.

implications for future measurements and modeling. First, the longitude-separated multipoint measurements, supported by the OMNI data base mid-point measurements at L1, are qualitatively consistent with the idea of corotation of quasi-steady solar wind structures at this quiet time of the solar cycle over a heliolongitude baseline of at least up to $45^{\circ}$. This justifies the use of the STEREO-B spacecraft for L1 solar wind prediction purposes (e.g. Biesecker, Webb, and St. Cyr, 2008), although the increasing separation, coupled with the expected increase in speed of the solar magnetic field evolution on the rising phase of cycle 24 will eventually modify this conclusion. The model comparisons on the other hand suggest that ecliptic solar wind source mappings at this time are sensitive to the accuracy of the polar field corrections used in the model synoptic maps. For the early portion of 2007 , when the source mappings were mainly to large southern hemisphere midlatitude holes, the WSA model of the stream structures and speeds agreed with the observations better than in the later portion of 2007, when the polar hole edge sources dominated the model mappings. The PFSS and WSA models were used to suggest a possible role played by the unusually 


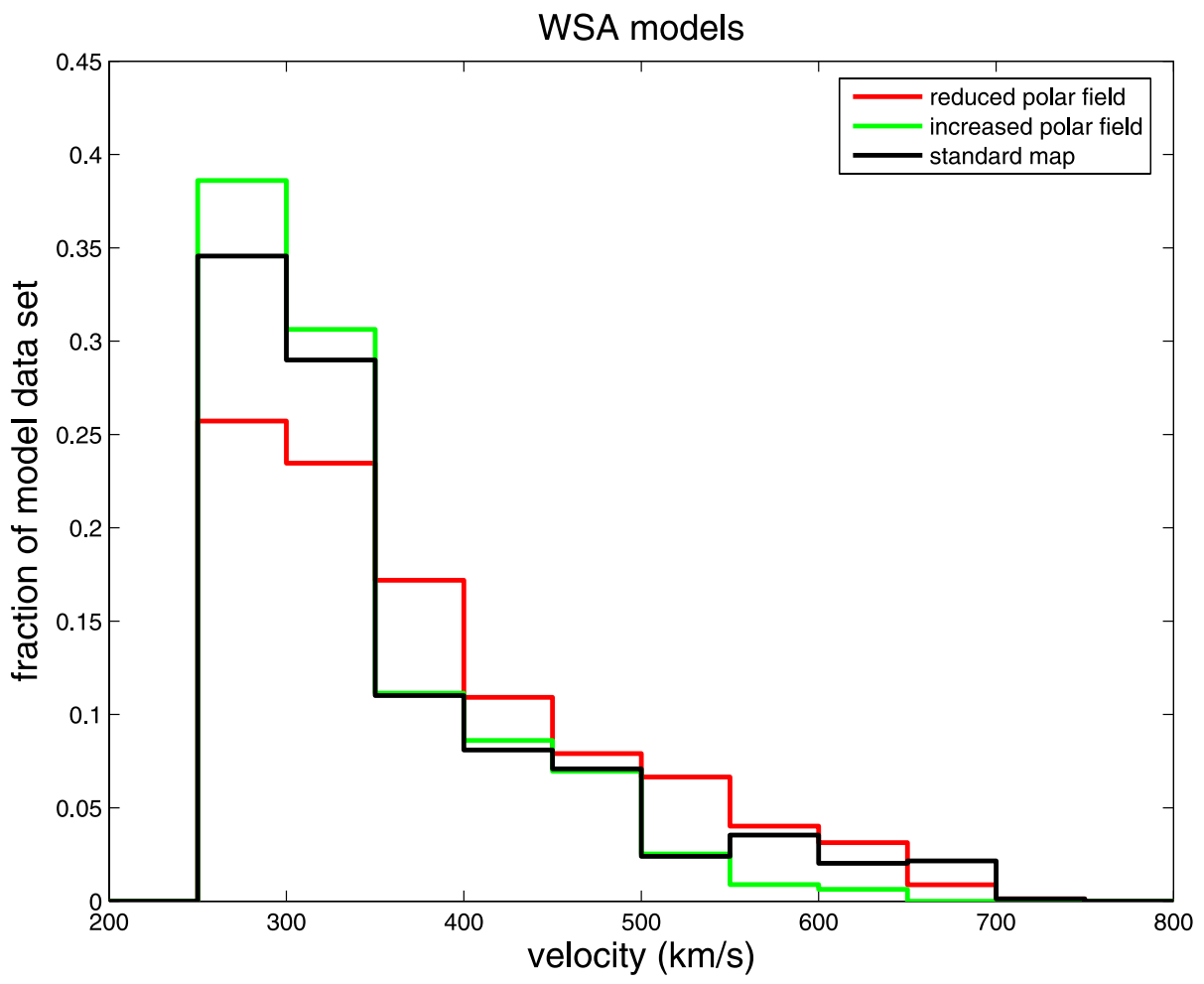

Figure 11 Calculated WSA model velocities based on the standard polar field treatments (black), compared to a reduced polar field model (red, see text), and an increased polar field model (green). The observed trend toward a flatter velocity distribution this cycle is seen in the model results with the weaker polar field.

weak solar polar fields. In particular, the models suggest that the observed persistent excess of high speed winds this cycle may be due in part to the weak polar fields. These results emphasize the importance of knowing the Sun's polar fields for understanding and predicting the solar wind. Finally, they illustrate one important way in which the solar dynamo measurably affects the quiet solar wind.

Acknowledgements This work was supported primarily by NASA contract NAS5-03131 to UCB for the STEREO IMPACT investigation and NSF agreement ATM-0120950 to the Center for Space Weather Modeling. The work shown here heavily involves the PLASTIC investigation solar wind ion measurements, provided by the PI (coauthor A.B. Galvin) and the PLASTIC team. The STEREO images shown are from SECCHI EUVI investigation and the STEREO Science Center at GSFC. We are grateful to the other contributing mission PIs and to the NSSDC and CDAWweb for constructing and providing the OMNI L1 in situ data set, and to the SOHO mission investigators and ESA for the open access to SOHO solar images. Finally, the modeling work could not have been carried out without the availability of ground-based magnetograms from Mt. Wilson Observatory and NSO GONG, which are jointly sponsored by the National Science Foundation, NASA and the US Air Force. We thank the observatories for providing open access to their observations and useful data products.

Open Access This article is distributed under the terms of the Creative Commons Attribution Noncommercial License which permits any noncommercial use, distribution, and reproduction in any medium, provided the original author(s) and source are credited. 


\section{References}

Altschuler, M.D., Newkirk, G. Jr.: 1969, Solar Phys. 9, 131.

Arge, C.N., Pizzo, V.J.: 2000, J. Geophys. Res. 105, 10465.

Baker, D.N., Li, X., Turner, N., Allen, J.H., Bargatze, L.F., Blake, J.B., Sheldon, R.B., et al.: 1997, J. Geophys. Res. 102, 14141.

Biesecker, D.A., Webb, D.F., St. Cyr, O.C.: 2008, Space Sci. Rev. 136, 45.

Delaboudiniere, J.-P., Stern, R.A., Maucherat, A., Portier-Fozzani, F., Neupert, W.M., Gurman, J.B., et al.: 1997, Adv. Space Res. 20, 2231.

Galvin, A.B., Kistler, L.M., Popecki, M.A., Farrugia, C.J., Simunac, K.D.C., Ellis, L., et al.: 2008, Space Sci. Rev. 136, 437.

Gosling, J.T., Asbridge, J.R., Bame, S.J., Feldman, W.C.: 1978, J. Geophys. Res. 83, 1401.

Hathaway, D.H.: 2008, Space Sci. Rev. doi:10.1007/s11214-008-9430-4.

Hoeksema, J.T., Scherrer, P.H.: 1984, Bull. Am. Astron. Soc. 16, 452.

Hoeksema, J.T., Wilcox, J.M., Scherrer, P.H.: 1983, J. Geophys. Res. 88, 1910.

Howard, R.A., Moses, J.D., Vourlidas, A., Newmark, J.S., Socker, D.G., Plunkett, S.P., et al.: 2008, Space Sci. Rev. 136, 67.

Kilpua, E.K.J., Luhmann, J.G., Gosling, J., Li, Y., Elliott, H., Russell, C.T., Jian, L., et al.: 2009, Solar Phys. this issue.

King, J.H., Papatashvilii, N.E.: 1994, Interplanetary Medium Data Book Supplement 5, 1988 - 1993, NASA NSSDC/WDC-A-R\&S 94-08, Greenbelt.

Lee, C.O., Luhmann, J.G., Odstrcil, D., MacNeice, P.J., de Pater, I., Riley, P., Arge, C.N.: 2008, Solar Phys. 254, 155.

Lee, C.O., Luhmann, J.G., Zhao, X.P., Liu, Y., Riley, P., Arge, C.N., Russell, C.T., De Pater, I., et al.: 2009, Solar Phys. in press.

Li, Y., Lynch, B.J., Stenborg, G., Luhmann, J.G., Huttunen, K.E.J., Welsch, B.T., Liewer, P.C., Vourlidas, A.: 2008, Astrophys. J. 681, L37.

Luhmann, J.G., Li, Y., Arge, C.N., Gazis, P.R., Ulrich, R.: 2002, J. Geophys. Res. 107, doi:10.1029/2001JA007550.

Luhmann, J.G., Curtis, D.W., Schroeder, P., McCauley, J., Lin, R.P., Larson, D.E., et al.: 2008, Space Sci. Rev. 136, 117.

McComas, D.J., Bame, S.J., Barker, P., Feldman, W.C., Phillips, J.L., Riley, P., Griffee, J.W.: 1998, Space Sci. Rev. 86, 563.

McComas, D.J., Ebert, R.W., Elliott, H.A., Goldstein, B.E., Gosling, J.T., Schwadron, N.A., Skoug, R.M.: 2008, Geophys. Res. Lett. 35. doi:10.1029/2008GL034896, CiteID L18103.

Ogilvie, K.W., Chornay, D.J., Fritzenreiter, R.J., Hunsaker, F., Keller, J., Lobell, J., et al.: 1995, Space Sci. Rev. 71, 55.

Pneuman, G.W., Kopp, R.A.: 1971, Solar Phys. 18, 258.

Schatten, K.: 1999, In: Habbal, S.R., Esser, R., Hollweg, J.V., Isenberg, P.A. (eds.) Solar Wind Nine, AIP Conf. Proc. 471, 409.

Schrijver, C.J., Liu, Y.: 2008, Solar Phys. 252, 19.

Simunac, K.D.C., Kistler, L.M., Galvin, A.B., Lee, M.A., Popecki, M.A., Farrugia, C., et al.: 2009, Solar Phys. submitted.

Smith, E.J., Balogh, A.: 2008, Geophys. Res. Lett. 35. doi:10.1029/2008GL035345.

Smith, C.W., L'Heureux, J., Ness, N.F., Acuña, M.H., Burlaga, L.F., Scheifele, J.: 1998, Space Sci. Rev. 86, 613.

Wang, Y.M., Sheeley, N.R. Jr.: 1990a, Astrophys. J. 355, 726.

Wang, Y.M., Sheeley, N.R. Jr.: 1990b, Astrophys. J. 365, 372.

Wang, Y.M., Sheeley, N.R. Jr., Rich, N.B.: 2007, Astrophys. J. 658, 1340. 